\title{
POSITION, LOCATION, PLACE AND AREA: AN INDOOR PERSPECTIVE
}

\author{
George Sithole ${ }^{a^{*}}$, Sisi Zlatanova ${ }^{\mathrm{b}}$ \\ ${ }^{a}$ Geomatics Division, School of Architecture, Planning and Geomatics, University of Cape Town, Private Bag X3, Rondebosch, \\ 7701, South Africa, Email: george.sithole@uct.ac.za \\ b 3D Geoinformation, Faculty of Architecture and the Built Environment, Delft University of Technology, Julianalaan 134, 2628 BL \\ Delft, The Netherlands, Email: s.zlatanova@tudelft.nl
}

\section{Commission IV/WG IV/7}

KEY WORDS: Indoor, Mapping, Modelling, Navigation, Application

\begin{abstract}
:
Over the last decade, harnessing the commercial potential of smart mobile devices in indoor environments has spurred interest in indoor mapping and navigation. Users experience indoor environments differently. For this reason navigational models have to be designed to adapt to a user's personality, and to reflect as many cognitive maps as possible. This paper presents an extension of a previously proposed framework. In this extension the notion of placement is accounted for, thereby enabling one aspect of the "personalised indoor experience'. In the paper, firstly referential expressions are used as a tool to discuss the different ways of thinking of placement within indoor spaces. Next, placement is expressed in terms of the concept of Position, Location, Place and Area. Finally, the previously proposed framework is extended to include these concepts of placement. An example is provided of the use of the extended framework.
\end{abstract}

Notable characteristics of the framework are: (1) Sub-spaces, resources and agents can simultaneously possess different types of placement, e.g., a person in a room can have an xyz position and a location defined by the room number. While these entities can simultaneously have different forms of placement, only one is dominant. (2) Sub-spaces, resources and agents are capable of possessing modifiers that alter their access and usage. (3) Sub-spaces inherit the modifiers of the resources or agents contained in them. (4) Unlike conventional navigational models which treat resources and obstacles as different types of entities, in the proposed framework there are only resources and whether a resource is an obstacle is determined by a modifier that determines whether a user can access the resource. The power of the framework is that it blends the geometry and topology of space, the influence of human activity within subspaces together with the different notions of placement in a way that is simple and yet very flexible.

\section{INTRODUCTION}

In recent years the mapping of enclosed environments has driven researchers to develop novel methods of representing these environments. These representations are used in a variety of applications, one of the most important applications being the assisted navigation of indoor spaces.

A shortcoming of conventional indoor navigation aids is that they tend to be very functional and mostly designed to answer the basic question of 'What is the best way to get from A to B?'. The emphasis has been on solving the technical problem of navigation at the expense of resolving human needs and expectations. One consequence of this is that navigational aids simplify the full range of human actions and conceptions during a navigation and focus on providing natural language directives, e.g., Chen and Mooney 2011.

This paper concerns itself with the more fundamental problem of how to model the dynamic interactions within indoor spaces, and specifically defining the elements that are required to model interactions within indoor spaces. Multi-Agent Systems (Van der Hoek and Wooldridge, 2008) have been used to model dynamic interactions in spaces and the work here will draw from work done in this area.

The authors previously proposed a framework (Zlatanova et. al. 2013) for modelling dynamics and navigation within indoor spaces. Others such as Goetz and Zipf, 2011, Khan and Kolbe,
2013, Jung and Lee, 2015, Yang and Worboys, 2015 have proposed similar models. While closer to the human perception of space, these models have a weakness in that they do not discuss the way humans perceive placement (how a thing occupies space), i.e., the conception of 'Here' and 'There'.

In contemporary navigational systems, travel between a start point and destination are imagined to be movements between pin points in space. But as humans we seldom think in this way when travelling. Our conception of 'Here' and 'There' is more complex. For example indoors a person goes from room 1.1, to the second floor, to room 2.3, and stops at a photocopier. During this travel our conception of 'Here' and 'There' is expanded (floor) and contracted (photocopier) in response to the context of the different parts of the journey. This paper looks at how best refer to this changing notion of 'Here' and 'There' into the model of an indoor environment.

Today many internet mapping services offer a richer notion of 'Here' and 'There' (e.g. Google Maps, Bing Maps, MapQuest, Open Street Maps, etc.). They achieve this by disambiguating between the geographical notions and moving from position to place (Hightower, 2003). In everyday conversation these words are used interchangeably, but as will be shown subtle differences exist between the words. The solution the authors propose is to build these notions of 'Position', 'Location', 'Place' and 'Area' into their indoor navigation framework.

\footnotetext{
* Corresponding author
} 
The paper is divided into three parts. Part one reviews the notions of 'Position', 'Location', 'Place' and 'Area'. Part two explores, with the use of examples, how these notions translate in indoor environments. Finally, part three proposes the inclusion of these notions into an indoor modelling framework previously developed by the authors.

\section{PART I}

\section{OVERVIEW: POSITION, LOCATION, PLACE, AND} AREA

In linguistics, referential expressions are those expressions that describe the placement of persons, objects and things. These aspects have been partially addressed by a large number of scientists dealing with defining relationships between objects. Most of the investigated expressions are between two objects, i.e. binary (I am at from of the coffee machine) and very few can be ternary (I am between the restaurant and the office of Tom). Therefore, most of the research has been focussed on identifying binary relationships. Three major approaches have been identified for representing spatial relationships namely metric, topology and direction (Kainz, 2010). The first sound models for representing spatial relationships have been based on point-set theory and the notion of boundary, interior and closure of point sets (Pullar and Egenhofer 1998, Herring 1991). This framework has been extended to cover 3D space and objects of different dimensions (Egenhofer and Herring, 1991, Zlatanova 2000). Topology represents neighbourhood relationships between two objects, which allows the definition of inclusion ('in') and closeness (meet, or next to). It's a powerful method to control impossible cases such as trees penetrating a house $(\mathrm{Xu}$ and Zlatanova 2013, Louwsma et al 2006) or mother holding the hand of a girl being located by a localisation system in one room and the girl in another. Many linguistic expressions such as 'at back', 'at front', or 'between' have been formally defined under the category of directional relationships. Directional relationships are more complex and require a specific frame of reference and/or context. Retz-Schmidt, 1988 discusses three frames of reference namely intrinsic, deictic and extrinsic. Intrinsic frame relies on providing relations with respect to the reference objects. An example of an intrinsic frame is 'right', 'left', 'at front of' the desk (the reference object). Frank 1998 introduces Euclidian frame of reference to make the directional relationships unambiguous. Another approach is to use the context of the situation. For example, if a person is at front of a desk, it is trivial to indicate what is left or right. Clementini, 2013 provides a formalisation of number of directional relationships by mapping them to projective relationships.

Referential expressions are used here to nuance the notion of placement in space. Because users of navigational aids will conceive and express their placement in space using natural language, it is reasonable to begin a study of placement by studying commonly used referential expressions.

\subsection{Prepositions}

Prepositions are words commonly used to indicate placement. Retz-Smith, 1988 provides an extensive review on the spatial prepositions used for location outdoors. Kray at al 2013 investigate the prepositions used in outdoor, indoor and transitional spaces. They conclude that some prepositions are used only for indoor or outdoor spaces. For example, the preposition 'through' was found to be the most used for all three types of spaces, while 'beneath' and 'behind' has been predominantly used indoors. Their study shows that humans use most spatial expressions within indoor space. Coventry et al 2005 proved that the prepositions depend on the object used for the reference.

We use the prepositions to investigate the notion of placement. Table 1 lists prepositions most commonly used in expressing placement or routes in/through space.

\begin{tabular}{|lllll|}
\hline Above & Beneath & In & Out & Underneath \\
Across & Beside & Inside & Outside & Until \\
Against & Between & Into & Over & Up \\
Ahead & Beyond & Left & past & Upon \\
Along & By & Near & Right & Up to \\
Around & Close by & Next & Through & Within \\
At & Down & Off & Towards & \\
Among & From & On (On top & To & \\
Behind & From...to & of) & Under & \\
Below & Front & Opposite & & \\
\hline
\end{tabular}

Table 1. List of common prepositions used in referential expressions

The rule for forming a prepositional phrase is thus:

\section{Preposition - Optional Modifier - Noun, Pronoun}

Table 2 provides examples of prepositional phrases. Because the rule contains nouns, pronouns, etc., an implication of the rule for forming prepositional phrases is that placement is with reference to space or other objects or things in space. In other words expressions of placement are relative.

\begin{tabular}{|l|l|l|l|}
\hline Preposition & Modifier & $\begin{array}{c}\text { Noun, } \\
\text { Pronoun, etc., }\end{array}$ & \multicolumn{1}{|c|}{ Example } \\
\hline Above & The & First floor & Above the first floor \\
\hline Along & The & Passageway & $\begin{array}{l}\text { Walk along the } \\
\text { passageway }\end{array}$ \\
\hline
\end{tabular}

Table 2. Examples of prepositional phrases

But there are seeming exceptions like the expression 'The toy store is at $33^{\circ} \mathrm{S}, 28^{\circ} \mathrm{E}$ ' doesn't refer to a noun or pronoun. Although the above expression doesn't reference a noun, pronoun, etc., the coordinates define a latitude and longitude within a geographical reference frame. In this expression the relative reference is implicit. Relative references that are fixed and agreed to by general consensus are called absolute references. This is to distinguish them from arbitrary relative references.

\subsection{Addressing}

Relative and absolute references can also be thought of addresses to placement in space. For indoor environments the addressing of sub-spaces is complex because (a) addresses will be building specific (no two buildings are guaranteed to use the same form of addressing), (b) the person navigating the building has to either have a cognitive map of the building (e.g., Moeser 1988) or be familiar with different spatial schemas of building floor plans. For example, the answer to the question 'Where are you in the building?' will depend on (a) the layout of the building, i.e., contextual knowledge, (b) how a person visualises themselves in the building. One person might say, 'At the photocopier' and another might say 'In room 2.13'. Although the answers may be different, if they refer to the same placement they are both legitimate. An indoor model has to be able to accommodate this ambiguity.

\subsection{Elements of referencing}

In classical geography the expression of the placement of objects, in relation to the outdoors, was given in terms of the concepts of 
Topos, Choros, and Geos (Mayhew, 2011). These concepts were typically distinguished by scale or specificity. Topos referring to the place, Choros refers to the region and Geos to the face of the earth. These concepts are mentioned here because not only can they be distinguished by scale, but also by the context of the environment, notions of 'belonging in space', and a person's conception of placement in space. This provides an avenue to understand and accommodate the ambiguity in addressing that is necessary for indoor models. Below are seven typical expressions of the same placement:

Statement 1: 'I am at $28.2314^{\circ},-33.4577^{\circ}$ '

Statement 2: 'I am on the chair'

Statement 3: 'I am next to the table'

Statement 5: 'I am in the living room'

Statement 6: 'I am at home'

Statement 7: 'I am in the neighbourhood'

From these statements certain aspects of addressing can be identified, referred to here as Reference, Specificity and Uncertainty, Scope and Context (see Table 3).

\begin{tabular}{|c|c|c|c|c|}
\hline & Position & Location & Place & Area \\
\hline Reference & $\begin{array}{l}\text { Absolute } \\
\text {, e.g., a } \\
\text { coordina } \\
\text { te } \\
\text { system }\end{array}$ & $\begin{array}{l}\text { Absolute, } \\
\text { e.g. room } \\
\text { number }\end{array}$ & $\begin{array}{l}\text { Relative, } \\
\text { Placement } \\
\text { in a room } \\
\text { (Inside) }\end{array}$ & $\begin{array}{l}\text { Relative, } \\
\text { Placemen } \\
\text { in an } \\
\text { aggregatio } \\
\text { n of rooms }\end{array}$ \\
\hline $\begin{array}{l}\text { Specificity/ } \\
\text { Uncertainty }\end{array}$ & $\begin{array}{l}\text { Depends } \\
\text { on the } \\
\text { device } \\
\text { providin } \\
\mathrm{g} \text { the } \\
\text { position }\end{array}$ & $\begin{array}{l}\text { Certain, } \\
\text { defined by } \\
\text { the } \\
\text { physical } \\
\text { borders } \\
\text { (walls) }\end{array}$ & $\begin{array}{l}\text { Uncertain, } \\
\text { defined by } \\
\text { the } \\
\text { functional } \\
\text { space of an } \\
\text { object, } \\
\text { e.g., Desk }\end{array}$ & $\begin{array}{l}\text { Uncertain } \\
\text { Defined by } \\
\text { a more } \\
\text { general } \\
\text { notations } \\
\text { (floors, } \\
\text { parts of } \\
\text { buildings) }\end{array}$ \\
\hline Scope & $\begin{array}{l}\text { Defined } \\
\text { by a } \\
\text { referenc } \\
\text { e frame }\end{array}$ & $\begin{array}{l}\text { Contains } \\
\text { Places }\end{array}$ & $\begin{array}{l}\text { Contained } \\
\text { in } \\
\text { Locations }\end{array}$ & $\begin{array}{l}\text { Contains } \\
\text { locations }\end{array}$ \\
\hline Context & $\begin{array}{l}\text { There is } \\
\text { no } \\
\text { context }\end{array}$ & Context & Context & Context \\
\hline Example & $\begin{array}{l}\text { I am at } \\
28.2314^{\circ} \\
33.4577^{\circ} \\
3\end{array}$ & $\begin{array}{l}\text { I am in the } \\
\text { living } \\
\text { room }\end{array}$ & $\begin{array}{l}\text { I am at the } \\
\text { photocopi } \\
\text { er }\end{array}$ & $\begin{array}{l}\text { I am on the } \\
\text { second } \\
\text { floor }\end{array}$ \\
\hline
\end{tabular}

Table 3. Position, Location, Place and Area

Reference: Statements 2 and 3 refer to placement relative to two different objects, i.e., a chair and a table. Here the spatial relationship is only defined by topology.

Specificity/Uncertainty: Statement 1 refers to a pin point placement in space. There is no uncertainty in the placement of the object in statement 1 . Here the spatial relationship is clearly metric. In contrast in statement 2 the definition of the extent has been expanded to an area about the chair. This introduces the notion of uncertainty which relates to the extent of the addressable space.

Scope: Statements 5, 6, and 7 provide the placement at the scale of a room, home and neighbourhood. Scope introduces the concept of the topological nesting of spaces, e.g. a room is inside a house and a house is inside neighbourhood.

Context: Statements 2, and 3 refer to the same placement but relative to a chair and a table. The choice of the reference here depends on the context in which the statement is being made. To understand this, consider that if context were irrelevant all would provide the same answer to a referential question (e.g., where are you?). Statement 1 is clearly without context.

From these aspects four conceptions of placement can be isolated: Position, Location, Place and Area (established concepts in Geography). The notions of Position, Location, Place, and Area in relation to the above four elements is summarised in Table 3. The next part looks at how the concept of Position, Location, Place and Area can be applied to indoor environments.

\section{PART II}

\section{AN INDOOR PERSPECTIVE OF POSITION, LOCATION, PLACE, AND AREA}

Figure 1 represents a simplified indoor scene within a building. The scene is intended to capture the most basic spatial navigation within an indoor environment, namely, movement between subspaces (rooms) and movement between floors. The scene also imagines that a building is divided into blocks and that each block contains one or more rooms. In the next sections the concepts of Position, Location, Place and Area will be explained in relation to this indoor scene.

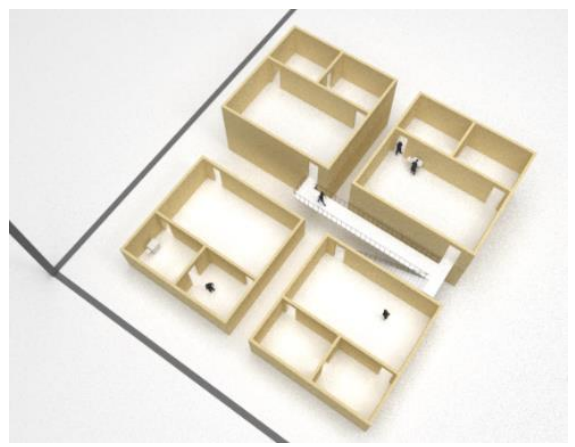

Figure 1. Indoor scene composed of 2 floors, 6 blocks ( 4 blocks on the ground floor), with each block containing 3 rooms. Two block on the ground floor and two blocks on the first floor are visible.

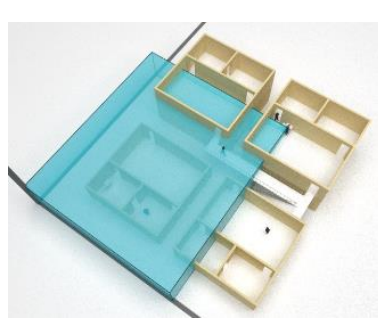

(a) Absolute position

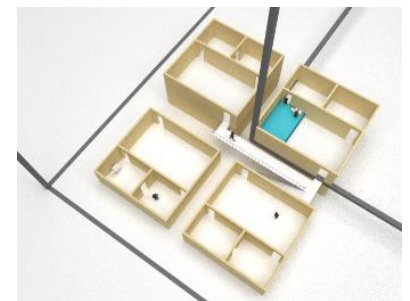

(b) Relative position
Figure 2. Position defines a pin point in space and it can be with reference to the origin of a global reference frame (left) or with reference to the origin of a local reference frame. The former and latter are referred to as absolute and relative positions respectively.

\subsection{Position}

A Cartesian reference system is defined for the building. This is shown by the grey lines in figure 2 . The absolute position of objects in the building are defined relative to this reference system. Note as discussed earlier, positions provide pin-point 
placements. Further reference systems can be defined for the blocks.

\subsection{Location}

Figure 3 shows the notion of location indoors. We specify a location referring to the smallest physically defined space in a building. These can be rooms, corridors, stairs, hall etc. Shown are people standing in room 2.2.A. The address of the room indicates the precise location of the room in the building. The address reads as 'second floor, second block, partition A'. It can be seen that room 2.2.A refers to a placement having some extent. Therefore, the exact Position of people in this room can't be inferred from the Location.

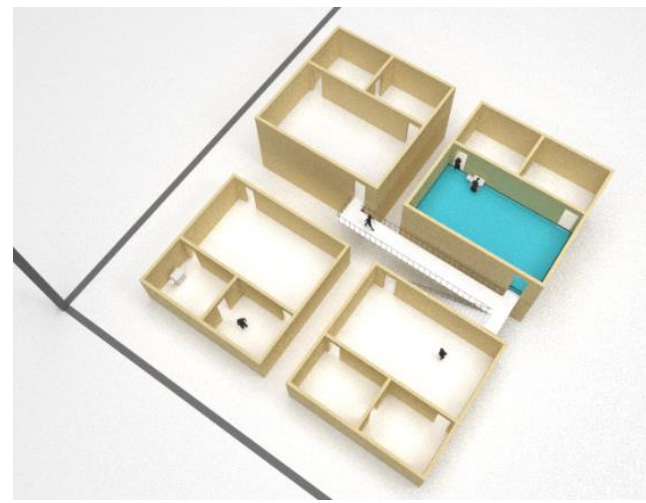

Figure 3. Location defines a general placement relative to welldefined physical space. For example in the figure the person can be said to be located in the room. The uncertainty in the position of the person is defined by the extent of the room. It should be noted that the speaker provides the context of the space.

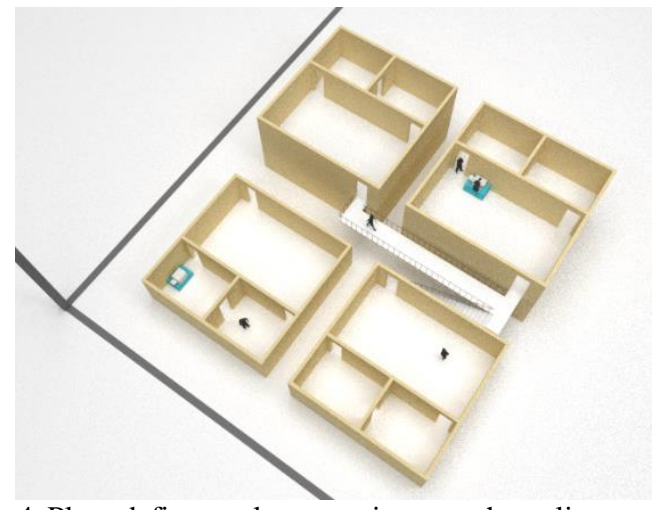

Figure 4. Place defines a placement in space that relies on a user's memory of space, i.e., the understanding of the layout of space is provided by the listener rather than the speaker.

\subsection{Place}

As shown in Table 3 Place refer to a particular object and the uncertain (functional) space around it. Place we use to indicate placements, which are within Locations and have uncertain or dynamic limits (Kruminaite and Zlatanova, 2014). Figure 4 shows an example of this. In this case Place is addressed as 'At the photocopier'. Because there are multiple photocopiers in the building the 'At the photocopier' expression references multiple placements. Which instance of 'photocopier' a user chooses to visit depends on either their cognitive map of the space or landmarks (Raubal and Winter, 2002, Richter, 2013) within the space. In any case if the specific placement has to be made explicit, a Location is needed.

\subsection{Area}

Area can be thought as of being a more generalised conception of Location. A helpful distinction between the two concepts is to think of an Area as containing multiple addressable locations. For example in figure 5 the Area is represented by the second floor, and the rooms on the second floor can be thought of as Locations. Though many aggregations, such a floors can be clearly identified, areas are most dependent on the cognitive map of a user and are source of misleading and confusion. Area placements are often used when the user is lost and seeks for more specific placement.

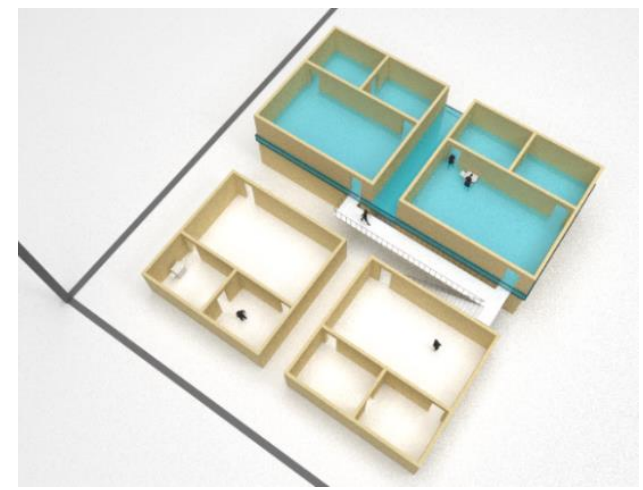

Figure 5. Area defines a generalised space or sub-space. In the figure the second floor represents an area. An Area is distinguishable from a Location in that and Area can be decomposed into Locations.

\section{NAVIGATION PERSPECTIVE}

In a multi-agent system the existence of agents and resources with different definitions of placement may be seen as an unnecessary complication of the navigation model. This would be the case if each agent or resource possessed a different form of placement, e.g., an agent whose placement is defined position and a resource whose placement is defined by Place. But this is not the intention here.

In the framework that will be explained in the next part, every entity (resource or agent) is simultaneously represented as a position, location, place and area.

\section{PART III}

\section{INDOOR NAVIGATION FRAMEWORK}

In 2013 the authors developed a framework to support indoor localization and navigation. The framework was developed around the subdivision of indoor spaces. This framework (shown in figure 7) is composed of Agents, Resources, sub-spaces, Modifiers and Activities.

\subsection{Conceptual framework}

Agents are those entities that navigate space. Space represents the entirety of the enclosed environment to be navigated. To facilitate navigation space is divided into sub-spaces. These sub-spaces can either be Free or Inert. Inert spaces are inaccessible by Agents. Examples of inert spaces are walls, indoor fountains and so forth. Free spaces (a) allow agents to move through them, (b) they contain resources, and (c) they host activities. 
In a navigation event an agent moves between free sub-spaces and either accesses resources or engages in activities. In figure 6 the agent moves between three sub-spaces and accesses the resource, Resource 3, residing in the last free space. An agent's use or access of a sub-space can be encumbered. For example a fire in a room (the sub-space) can render the room temporarily unusable. Modifiers are used to define the environment of a subspace. Sub-spaces can be encumbered by multiple modifiers. For example modifiers can be defined for lighting, temperature, sound and so forth. Together with the character of the agent (including the tasks that need to be performed), modifiers will determine the activities that are possible in the sub-space. For example if the elevator is broken (i.e. the status of resource is modified) a person carrying cleaning equipment that performs activity 'Cleaning' is not possible to go to the second floor to complete the activity. In this way the framework is able to simultaneously accommodate multiple agents with variable needs.

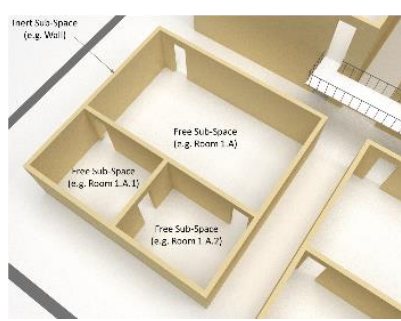

Free and inert Spaces

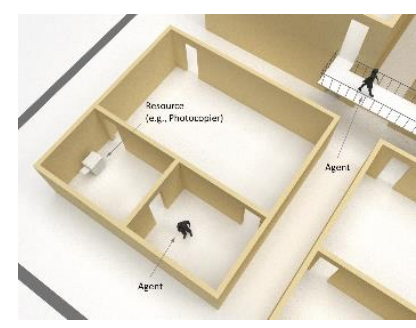

Agents and Resources

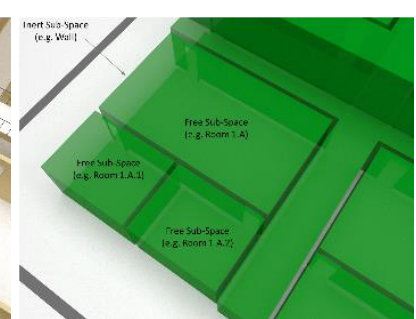

The dual of free and inert spaces

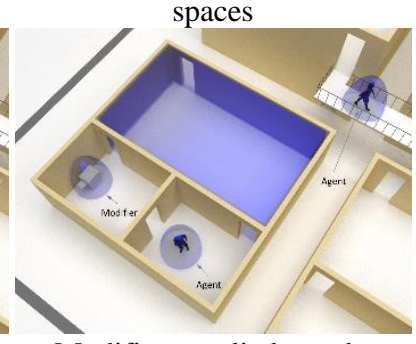

Modifiers applied to subspaces, agents and resources
Figure 6. The proposed framework. Sub-spaces are divided into free and inert spaces. Sub-spaces are occupied by resources. Subspaces are also occupied by agents who perform activities in the sub-space or access resources. All sub-spaces, resources and agents possess the property 'Placement' that defines where the entity is in space.

\subsection{Updated conceptual framework}

The 2013 framework sought to accommodate customisable navigation by multiple agents, i.e., the navigation route for any two agents with different intentions is not guaranteed to be the same. While the framework was designed to be as flexible as possible the way in which agents, resources and activities were referenced remained the same for all, and it was typically given as a position. As already mentioned this reduces the richness of navigational instructions.

In figure 7 placement is augmented by the concepts of position, location, place and area. In this conception every sub-space simultaneously possesses a position, location, place and area quartet. This change allows routes through space to be defined as in section 4.1. Notably in this conception an agent is allowed to enter a sub-space with one address and leave it with an alternate address. This refinement is necessary because (a) our activities in a sub-space can alter our perception of that sub-space, and (b) we enter spaces searching for resources at specific positions locations, or places, but we leave areas.

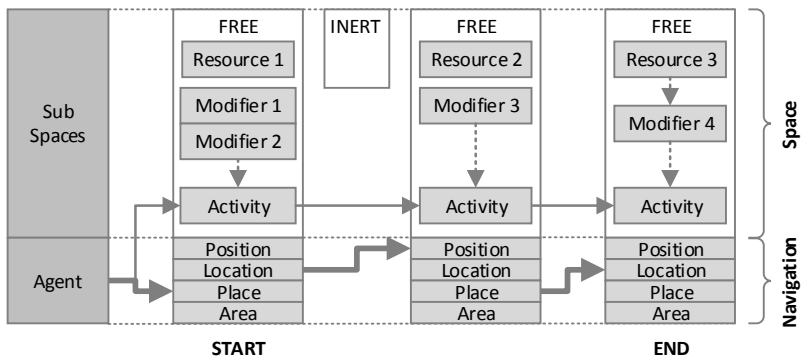

Figure 7. Position, Location, Place and Area built into the framework.

An example of this expansion/contraction of an address is show in figure 8. The agent has to travel from Room 2.A.3 to Room 1.B.2. The route can be addressed as Room2.A.3, Block A, Floor 2, Floor 1, Block B, Room 1.B.2.

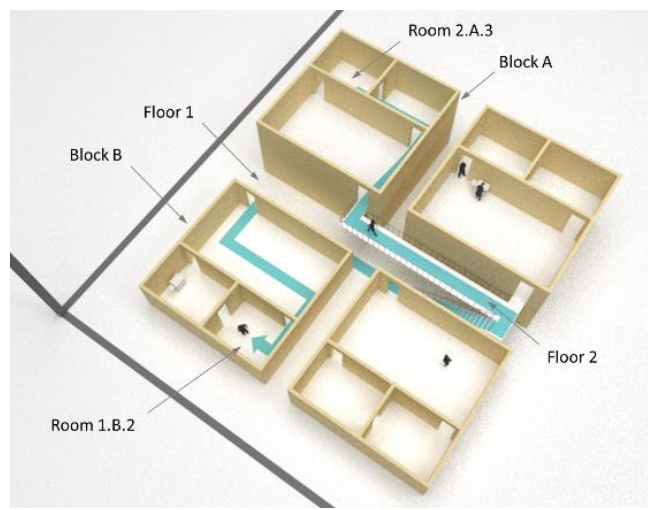

Figure 8. Expansion vs Contraction of an address. As the agent moves between the sub-spaces to reach Room 1.B.2 his/her address inflates and then deflates.

\subsection{Logical framework}

Indoor navigation systems are built on top of partition-maps (or subdivision maps) of indoor spaces. These partition-maps are obtained by subdividing indoor spaces such that they reflect cognitive spatial schemas (e.g. Khan and Kolbe 2013) of most users (agents), e.g., rooms, corridors, stairways and so forth. Various subdivision models have been proposed (Afyouni et al, 2012, Zlatanova et al 2014). The two most popular variants are tessellations and networks. This proposed framework is indented to be universal and does not depend on the type of subdivision. The remainder of this section will demonstrate the utility of the proposed navigation framework for a network model. Applications to tessellation models will be discussed in future papers.

Network models: Figure 9 shows the test indoor space represented as a network. The subdivisions (e.g., rooms, stairs, etc.,) are represented by nodes, V, and the topological relationship between the nodes is captured by the edges, E. The nodes and edges are commonly stored in a graph structure, $G(V$, E).

Dominant and auxiliary traits: Each node in the graph has a placement $\mathrm{P}$. While every node simultaneously possesses all four types of placements (position, location, place and area), some nodes have a greater affinity for specialisation than generalisation and vice versa. This affinity is here called the Dominant Trait. 
The alternative is called the Auxiliary Trait. For example in figure 9 it can be seen that the placement of Agents (e.g. people) and Resources (e.g., photocopier) are specific and will most likely be referred by positions or places. Position and Place are the dominant traits of the placement. Entrances and exits also exhibit this trait. On the other hand the placement of rooms is general in character and the dominant traits of placement are Location and Area.

Modifier influence inherited from the dominant trait: Each node in the graph also has the attribute $\mathrm{M}$, representing the set of modifiers that apply to a placement. Here again the spatial specificity of the modifier inherits from the dominant trait of the placement. For example a fire (the modifier) in room 2.A.3 is conceptualised to encompass the whole of the room. However, a more specific placement can be inferred from a fire at a photocopier. This inheritance mechanism provides a more natural expansion and contraction of the influence of modifiers.

The navigation process: A guided navigation consists of three main activities, planning (path computation), giving instructions, receiving feedback (either by the user or by a localisation system) and correcting the path if needed (Goetz and Zipf 2011, Zlatanova et al 2014). The planning phase aims to determine the least cost route between two or more points contained in the space. This cost is typically measured in terms of distance, time and risk. The giving and receiving instructions phase determines how best to verbally ask for a route and how best to verbally convey the route to the asker. The travelling phase considers the movement between the source and destination and how the agent is assisted during the movement.

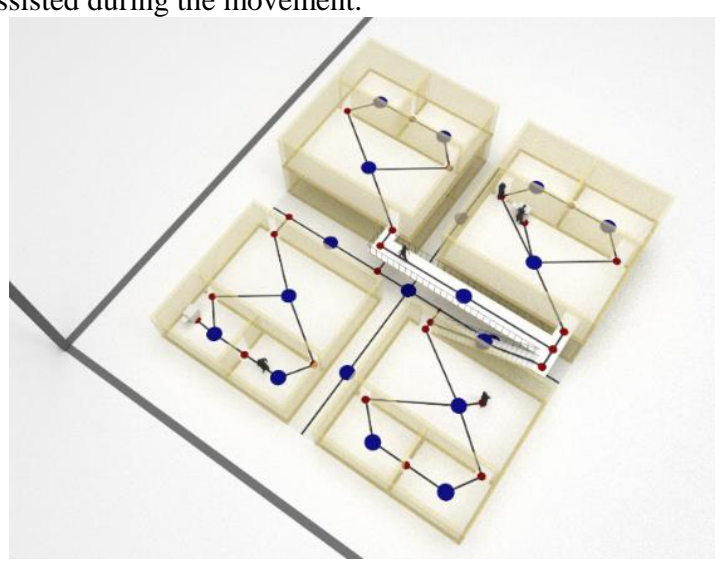

Figure 9 Representation of indoor space as a network. The large blue circles represent locations indoors, they reference general placement. The smaller red circles represent positions or places, and reference specific placement. All placements are assigned positions.

The selected route determined from the navigation process can be described as $G_{\text {Route }}\left(\mathrm{V}_{\text {position/cost}}, \mathrm{E}_{\text {cost }}\right)$ where GRoute is a subgraph of G. Note that in conventional solutions vertices are only attributed by a position and the cost of passing through a vertex. The best route is then identified as that route for which optimising function $\Phi$ is given by:

$$
\Phi=\Sigma \mathrm{E}_{\mathrm{cost}}=\min
$$

Conventional indoor navigation models have various limitations. For example conventional indoor models do not account for dynamic environments (e.g., moveable resources), variable notions of placement (during travel) and that agents will navigate space differently. For this reason the proposed framework includes the concepts of agents and resources, and introduces the concepts of modifiers and placement. The choice of route is shown in figure 10. The navigation model is divided into three segments, the Environment segment, the Multi-Agent System segment and the Route segment. The Environment segment describes the subdivision of space and the placement of resources within the space. A union of the subdivision of space and the placement of resources yields the environment graph/network.

$$
\mathrm{GEnv}_{\mathrm{E}}=\mathrm{G} \text { space } \cup \mathrm{GRes}_{\operatorname{Res}} \cup \mathrm{G}_{\operatorname{Res} 2} \cup \ldots \cup \mathrm{G}_{\operatorname{Res} N}
$$

The benefit of this approach is that resources can be easily added/removed from the environment in real-time. An added benefit is that only those resources that are desired by an agent need be included in the environment model. The Multi-Agent System segment models the interaction of multiple agents within the environment. The proposed framework imagines that during a navigation an agent will access multiple resources and/or agents. Here the optimum route is determined by a function $\Phi$ over the environment graph and the graph of each agent in the space.

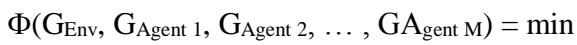

Unlike equation (1) the function $\Phi$ is non-trivial and dependent on the character of the agents at a particular moment in time. The content of the function $\Phi$ will be discussed in a future paper.

The function of Modifiers in $\Phi$ : In figure 10 it will be seen that all vertices, $\mathrm{V}$, and edges, E possess a modifier as an attribute. Furthermore the sub-spaces, resources and agents also possess modifiers. Attributing all elements of the framework with modifiers may seem overwrought but as will be show with examples it provides a powerful mechanism for both designing the multi-agent system and forming route instructions. The reader may have noticed that obstacle are missing in the framework as shown in figure 10. The reason for this is that depending on user perspective a resource can also be treated as an obstacle. For example for those who want to sit in a chair within a room a chair is a resource, but for those passing through a room it's an obstacle to be navigated. How a resource morphs into an obstacle is determined by the modifier associated with the resource. Therefore, an obstacle is a resource encumbered by a modifier that repels those who do not desire the resource. This provides a more faithful modelling of how agents perceive the world and interact with resources and obstacles. As can be seen modifiers are a powerful way of building dynamism into navigation models. In the next example the function of modifiers is taken even further.

Attributing agents with modifiers allows agents to influence the space they move in. For example when a security official enters a room he/she can impose restrictions on activities that may take place in that room. As indicated earlier modifiers inherit from the dominant trait of placement. This allows spaces, resources and agents to possess modifiers that are adapted to their various forms of placement, which permits rich interpretations. For example a resource, Res1, has the following placements, Position $=(23.12$, $50.2,45.1)$, Location $=$ 'Room 1.A.2', Place $=$ 'Photocopier', Area $={ }^{\text {'st }}$ Floor'. If Res1 is on fire, a modifier called 'Fire' can be associated with Res1. Naturally, the region of influence of the modifier depends on the form of placement and different definition of the 'Fire' modifier will have to be defined for each form of placement. The forms and interaction of placements and modifiers are complex. Theses interaction are modelled in the function $\Phi$. The form of modifiers cannot be elaborated in the 
space available here and will therefore be left for discussion in a future paper.

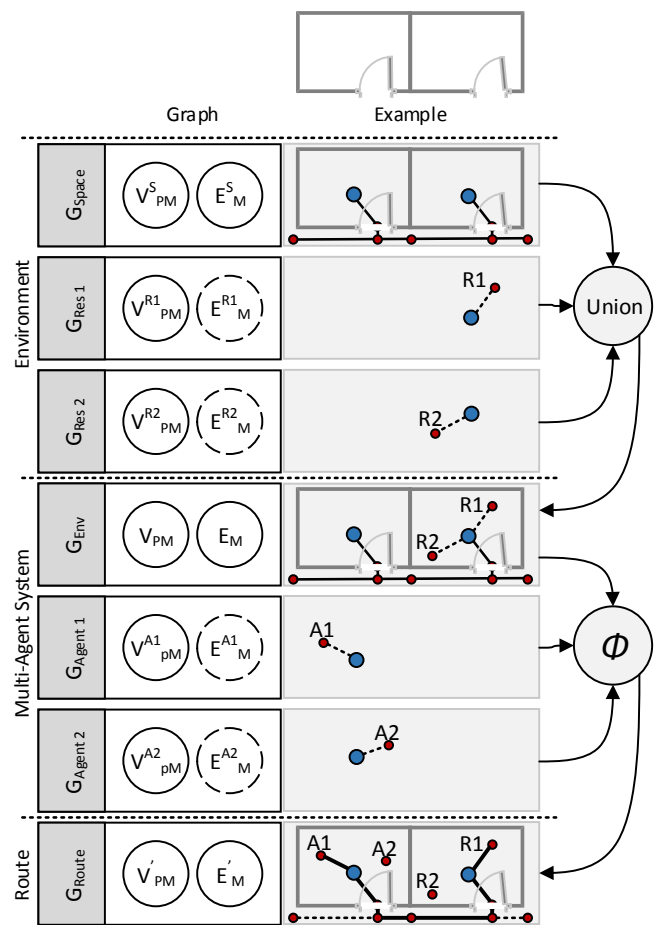

Figure 10. Representation of indoor space as a network. The large blue circles represent locations indoors, they reference general placement. The smaller red circles represent positions or places, and reference specific placement.

\subsection{Application of the Framework}

In this section an application of the framework is demonstrated using a simple example. The problem is composed of the following element shown in table 4.

\begin{tabular}{|l|l|l|l|}
\hline \multicolumn{1}{|c|}{ Item } & \multicolumn{1}{|c|}{ Type } & $\begin{array}{c}\text { Dominant } \\
\text { Trait }\end{array}$ & \multicolumn{1}{|c|}{ Modifier } \\
\hline Secretary & Agent & Position & Space Lock \\
\hline Cleaner & Agent & Position & Space Lock \\
\hline Photocopier & Resource & Place & Access 0 \\
\hline Floor 2 & Sub-Space & Area & \\
\hline Stairs & Sub-Space & Location & \\
\hline Floor 1 & Sub-Space & Area & \\
\hline Corridor & Sub-Space & Location & \\
\hline Room 1.A & Sub-Space & Location & Slippery Floor \\
\hline Room 1.A.1 & Sub-Space & Location & \\
\hline Room 1.A.2 & Sub-Space & Location & \\
\hline Table & Resource & Place & Access 1 \\
\hline Plant & Resource & Place & Access 1 \\
\hline
\end{tabular}

Table 4. A navigation problem demonstrating the application of the framework

Parameters of the problem: (1) The Secretary needs to use a photocopier in room 1.A.2. To use the copier the Secretary has to travel from Floor 2 (upper floor) to Floor 1 (lower floor) and reach Room 1.A.2 (Photocopier room). (2) The Cleaner is working on the ground floor. (3) The Secretary and Cleaner may not be in the same room at the same time. (4) When travelling through Room 1.A the Secretary has to navigate past a Table and Plant (obstacles). The Cleaner has to tend to the Table and Plant (resources). Here the Table and Plant possess dual roles. (5) Room 1.A has just been cleaned and the floors are slippery.

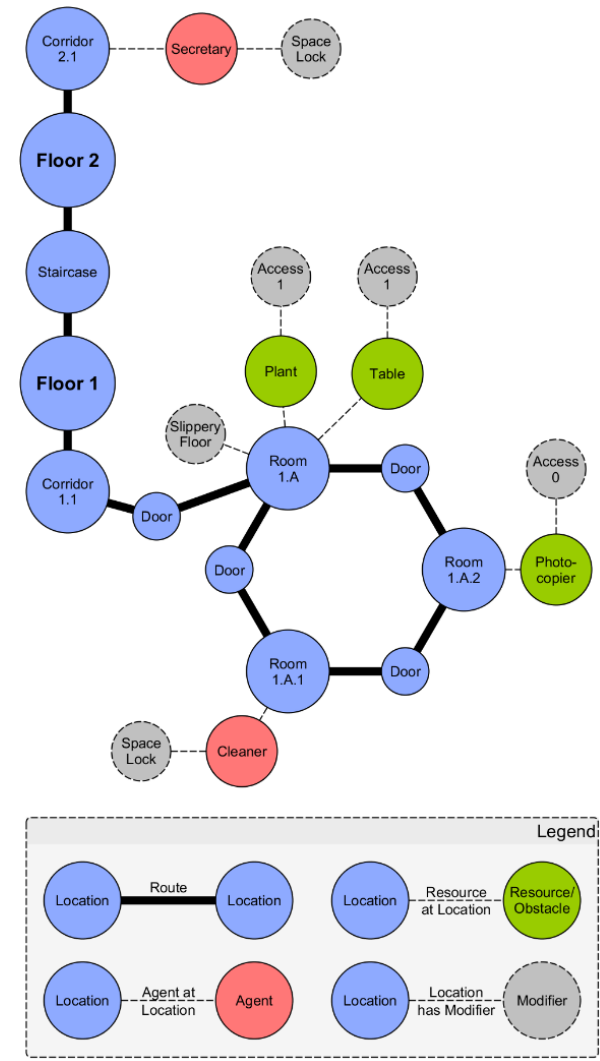

Figure 11 Application of the navigation framework. Blue circle represent entities (in this case sub-spaces) whose dominant trait is Location. Red circles represent entities (in this case agents) whose dominant trait is Position. Green circles represent entities whose dominant trait is Place.

Figure 11 shows the problem implemented according to the representation in figure 10. Note that the network in figure 11 is sub-network of the network shown in figure 9. The sub-spaces (shown in blue) are to be traversed by the Secretary who is in Corridor 2.1. Each sub-space inherits the modifiers of its attendant agent or resource/obstacle, e.g., Room 1.A.1 is inaccessible because this sub-space inherits the global Space Lock modifier of the Cleaner. To reach the photocopier the Secretary will have to travel through Room 1.A. Room 1.A has the global modifier Slippery Floor. Therefore, the Secretary will be cautioned when entering this room. Room 1.A. Room 1.A also inherits the local Access 1 modifier from the Plant and the Table. The Secretary doesn't have Access 1 permissions which causes the Plant and Table to become obstacles. Room 1.A.2 inherits the local Access 0 modifier from the photocopier. The Secretary has Access 0 permissions and because of this the photocopier becomes a resource.

\section{CONCLUSION}

This paper presents an extension of previously proposed framework on space subdivision, in which the notions of placement are accounted for. Placement is expressed in terms of the concepts of Position, Location, Place and Area. The power of the framework is that it blends the geometry and topology of space, the influence of human activity within sub-spaces together with the different conceptions of placement in a way that is simple and yet very flexible and intuitive. The proposed framework operates only with resources. Whether a resource is an obstacle is determined by a modifier that determines whether a user can access the resource. Furthermore, the expression for 
navigating around a resource (obstacle) is determined by the dominant placement of the resource.

The great benefit of the model is that it's scalable and permits the real time addition and removal of entities. Future work will identify the various forms of agents, resources, and modifiers. Importantly work will be done in determining rules for designing the optimising function $\Phi$ when searching for optimal routes within indoor environments.

\subsection{References}

Afyouni, I., Ray, C., and Claramunt, C. 2012. Spatial models for context-aware indoor navigation systems: A survey, Journal of Spatial Information Science, Number 4 (2012), 85-123.

Chen, D. L., and Mooney R. J., 2011. Learning to interpret natural language navigation instructions from observations. Learning to interpret natural language navigation instructions from observations. In Association for the Advancement of Artificial Intelligence (AAAI), pp 128-135, Cambridge, MA.

Clementini, E, 2013. Directional relations and frames of reference. GeoInformatica, 17(2). pp 235-255

Coventry, K. R., A. Cangelosi, R. Rajapakse, A. Bacon, S. Newstead, D. Joyce, L. V. Richards, 2005. Spatial Prepositions and Vague Quantifiers: Implementing the Functional Geometric Framework, Spatial Cognition IV. Reasoning, Action, Interaction, Vol. 3343, Lecture Notes in Computer Science pp. 98-110

Egenhofer MJ, Herring JR, 1991. Categorising topological relations between regions, lines and points in geographic databases, in: Egenhofer MJ and Herring IR (Eds.), A Framework for the Definition of Topological Relationships and an Approach to Spatial Reasoning within this Framework, Santa Barbara, CA. pp $1-28$

Frank, A.U., 1998. Formal models for cognition-taxonomy of spatial location description and frames of reference, Spatial Cognition, LNCS, Springer, pp. 293-312

Goetz, M., and Zipf, A. 2011. Formal definition of a useradaptive and length-optimal routing graph for complex indoor environments. Geo-Spatial Information Science, 14(2), 119-128

Hightower, J., 2003. From Position to Place. Proceedings of the 2003 Workshop on Location-Aware Computing. Fifth International Conference on Ubiquitous Computing, October 1215, 2003,

Jung, H. and Lee, J.: Indoor subspacing to implement IndoorGML for indoor navigation, Int. Arch. Photogramm. Remote Sens. Spatial Inf. Sci., XL-2/W4, 25-27.

Kanz, W, 2010, The mathematics of GIS, Department of Geography and Regional Research, University of Vienna, Vienna, Austria.

Khan, A. A. and Kolbe, T. H., 2013, Subspacing, based on connected opening spaces and for different ocomotion types using geometric and graph-based representation in multi-layered space-event model (MLSEM), ISPRS Ann. Photogramm. Remote Sens. Spatial Inf. Sci., II-2/W1, 173-185.

Kray, C., H. Fritze, T. Fechner, A. Schwering, R. Li, V. Joy Anact, 2013. Transitional Spaces: Between Indoor and Outdoor
Spaces, Spatial Information Theory. 11th International Conference, COSIT 2013, Scarborough, UK, September 2-6, 2013. Proceedings, Vol. 8116, LNCS. pp. 14-32

Kruminaite, M. and S. Zlatanova, 2014. Indoor Space Subdivision for Indoor Navigation. ISA'14, Proceedings of the Six ACM SIGSPATIAL International Workshop on Indoor Spatial Awareness, pp. 25-31.

Louwsma, J., S. Zlatanova, R van Lammeren, and P. van Oosterom, 2006. Specifications and implementations of constraints in GIS, In: GeoInformatica, Vol. 10(4). pp. 531-550

Mayhew, R., 2011, Geography's Genealogies, in Agnew and Livingstone (Eds.) The SAGA handbook of Geographical knowledge, SAGE, Los Angeles, pp. 21-38

Moeser, S. D., 1988. Cognitive mapping in a complex building. Environment and Behavior 20(1). pp 21-49.

Pullar D.V., Egenhofer, M.J., 1988. Toward the definition and use of topological relations among spatial objects. In Proc. Third Int. Symp. on Spatial Data Handling, pp. 225-242.

Raubal, M. and S. Winter, 2002, Enriching wayfinding instructions with local landmarks. Geographic information science, LNCS, Springer, pp. 243-259

Retz-Schmidt, G., 1988. Various views on spatial prepositions, AI magazine, 9(2), pp. 95-105

Richter, K-F., 2013, Prospects and challenges of landmarks in navigation services. In Cognitive and Linguistic Aspects of Geographic Space, pages 83-97. Springer, 2013.

Van der Hoek, W., and Wooldridge, M, 2008. Multi-agent systems. Foundations of Artificial Intelligence 3, pp 887-928.

Yang, Y., and M. Worboys, 2005, Generation of navigation graphs for indoor space, International Journal of Geographical Information Science, 29 (10) pp. 1737-1756

$\mathrm{Xu}, \mathrm{D}$. and S. Zlatanova, 2013. An approach to develop 3D GeoDBMS topological operators by reducing existing 2D operators, ISPRS Annals - Volume II-2/W1, 2013, WG II/2, 8th 3D GeoInfo Conference \& ISPRS WG II/2 Workshop, November 2013, Istanbul, Turkey. pp. 291-298

Zlatanova, S., 2000. On 3D topological relationships, In: Proceedings of the 11th International Workshop on Database and Expert System Applications (DEXA 2000), 6-8 September, Greenwich, London, UK. pp. 913-919

Zlatanova, S., Liu, L., and Sithole, G., 2013. A conceptual framework of space subdivision for indoor navigation. In The ISA '13 Proceedings of the Fifth ACM SIGSPATIAL International Workshop on Indoor Spatial Awareness, ACM, NY, USA, pp. 37-41.

Zlatanova, S., L. Liu, G. Sithole, J. Zhao and F. Mortari, 2014, Space subdivision for indoor applications, GISt Report, nr. 66, Delft, 201450 p. 